\title{
APPLICATION OF PIEZOCOMPOSITE TWIN, SIDE BY SIDE, PHASED ARRAY UT PROBES FOR THE INSPECTION OF STAINLESS STEEL
} M. Delaide ${ }^{1}$, and Ph Dumas ${ }^{2}$

${ }^{1}$ AVI International, Vilvoorde, Belgium, ${ }^{2}$ Imasonic SA, Besancon, France

\begin{abstract}
Ultrasonic examination of austenitic stainless steel components and welds takes an increasing place during the fabrication and the "in service inspection" of power and petrochemical plants, where cost reduction and severe safety rules impose to use innovative NDT methods with increased efficiency and flexibility.

As a consequence, the UT probes to be used must allow to detect and size cracks in coarse-grain structure, with a high reliability level while the duration of the examinations must be drastically reduced.

The combination of well-known TRL probes, based on twin side by side (emission / reception) crystals, with new technologies such as Phased array and piezocomposite allows for manufacturing new kind of transducers that improve both probes performances and inspection speed as requested.

Single element crystals are replaced by matrix arrays, making it possible to deflect and to skew the beams, to change the inspection depth and, by way of consequence, to reduce the probe displacement to a minimum.

Piezocomposite material improves the sensitivity and the cross coupling between elements thanks to its electro acoustical properties.

This paper describes the designing, the manufacturing and the characterisation of several different probes.

Application examples where these probes have been used will be presented.
\end{abstract}

Introduction: Last development in the field of UT on stainless steel components: The performance of ultrasonic examination techniques in stainless steel austenitic structures and welds, dissimilar metal welds and clad components are often strongly affected by the anisotropy and by the lack of homogeneity of the considered materials. The major problems encountered are beam skewing and distortion, high and variable attenuation, and high background noise.

Conventional UT techniques are less applicable on these materials because of the commonly very low signal to noise ratio achieved and because of the uncertainty about flaw location.

To overcome these difficulties, specific TRL (Transmitter Receiver L waves) ultrasonic probes have been successfully developed the last decade, presenting generally the following main characteristic: focussed low frequency compression waves, heavy damping, side by side transmitter receiver configuration.

Besides in the past 4 years, advanced TRLPA (Transmitter Receiver L waves Phased Array) design has been tested, leading naturally to the last version presented here: it is a combination using the versatility offered by the phased array technology with the intrinsic advantages of low-frequency TRL transducers, along with the high sensitivity, short pulses and the extended element cutting possibilities offered by the piezocomposite technology.

This new TRLPA piezocomposite design allows excellent examination performance, even on "challenging" inspection configurations where optimised UT inspection capability is needed in addition with a drastic reduction of the inspection time.

Piezo composite TRL PA principles and design: In conventional TRL search units, the apparent acoustic beam results from the convolution of the sound fields of the separate transmitter $(\mathrm{T})$ and receiver $(\mathrm{R})$ piezoelectric elements. A quasi-focusing effect in the beam-crossing region is obtained, substantially improving the signal-tonoise ratio in a limited depth range. Further more, the absence of "dead zone" allows for covering depth ranges starting from right under the scanning surface (1). The innovative TRLPA piezocomposite design showed in figure 1 adds the beam steering and beam focusing capabilities of a 3D matrix of elements on both $\mathrm{T}$ and $\mathrm{R}$ side: the beam can be skewed and focussed in a large area, presenting in all the cases optimised and controlled beam characteristics. Moreover, the high sensitivity of the piezocomposite material permits to work with low amplification, comfortably above the electronic noise, even in industrial environments

Side view Front view 

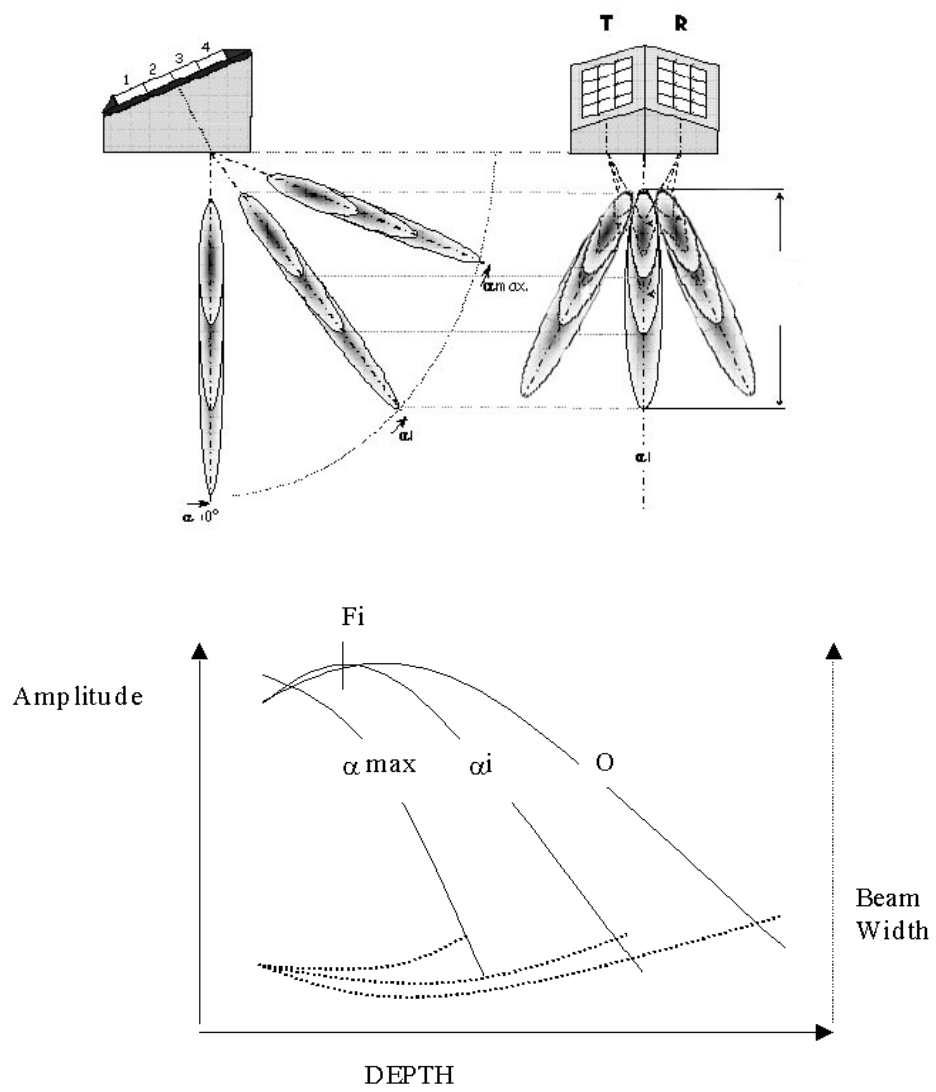

Figure 1

The TRLPA probe acts as an addition of virtual probes, presenting each individual sensitivity and beam width characteristics (curves in figure 1). In this way, specific design of the piezocomposite 3D matrix, makes it possible, if needed, to cover the whole thickness of a component, from the near surface to the bottom, in one scanning operation with one probe.

The design of TRLPA requires advanced methodologies and software to avoid secondary (grating) lobes and realise the necessary beam simulations. Figure 2 shows, as an example, the beam simulations of a $1 \mathrm{MHz}$ probe (dedicated to primary loops of PWR nuclear power plants) in the plane of incidence, where both L and SW beams are calculated, and in a perpendicularly section situated at the maximum of amplitude. One can notice the absence of side lobes and the beam symmetry. 


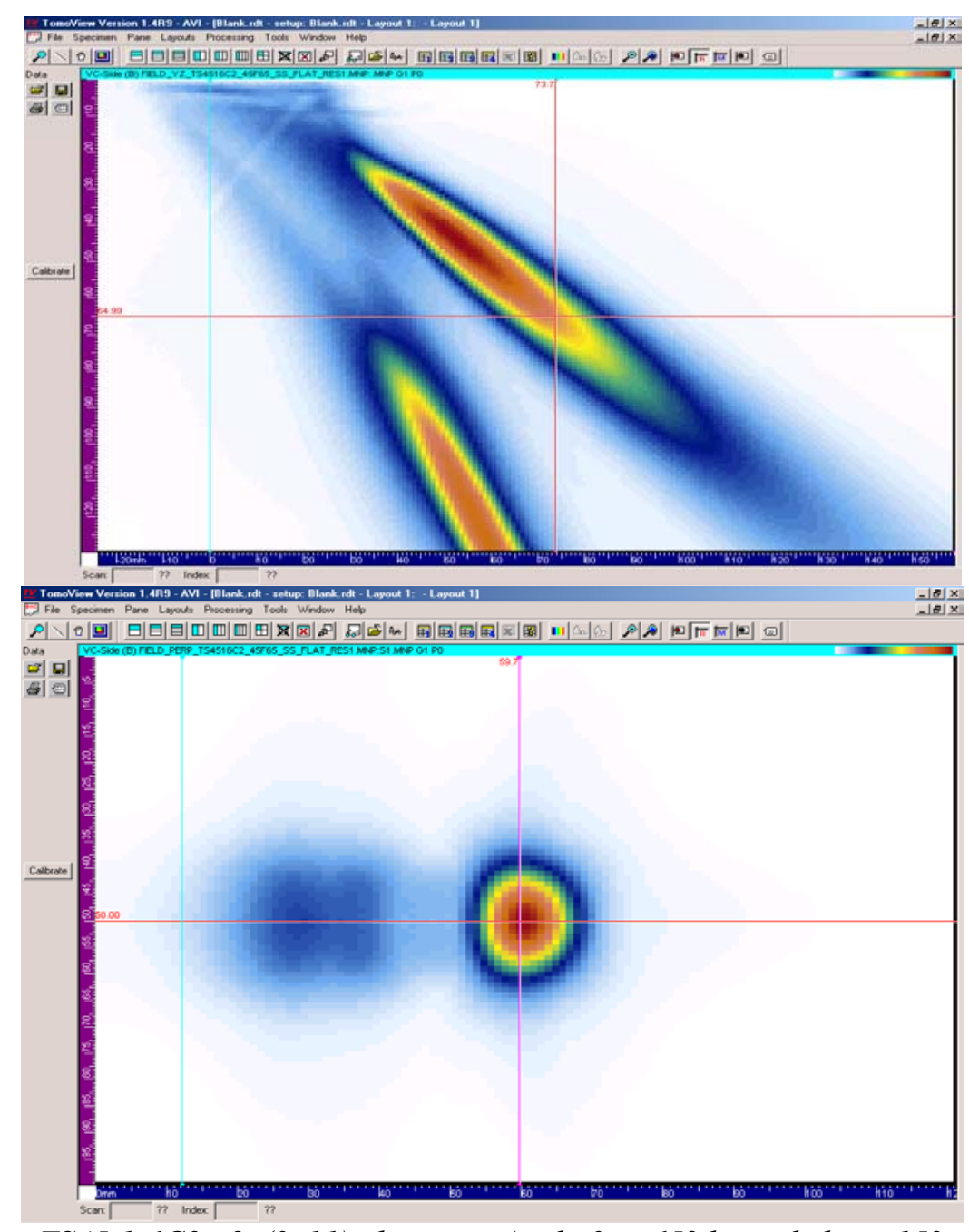

Probe Type :TS45 $16 C 2$, 2x(3x11) elements, Angle 0 to $65^{\circ}$,lateral skew $\pm 15^{\circ}$, foot print $54 x 54 \mathrm{~mm}$ Application: primary loops of PWR nuclear power plants, detection of thermal cracks

Figure 2

The focal laws, corresponding to the definition of the virtual probes generated by a TRLPA, are calculated by mean of specialised software (PASS, CIVA 7) or calculator routine integrated to UT acquisition and analysis software (Tomoview 2)

In addition, interesting features of TRLPA design useful to industrial applications, are to be mentioned: the possibility to generate, in complement to the active laws, a straight longitudinal beam allowing for coupling and thickness control and the possibility to avoid under certain circumstances wedge shaping.

\section{Electroacoustical designing:}

Probes are manufactured by Imasonic, based on specifications supplied by AVI.

The goal of this step is to defined the different components to use inside the transducer to achieve the performances level indicated in previously defined specifications.

The influence of these components on transducer performances is simulated using softwares based on KLM or finite elements.

The target parameters are temporal and spectral response, sensitivity and electrical impedance (see figure 2) Combined with these software, Imasonic database on Piezocomposite material allow precise simulation and very predictable electroacoustical performances. 

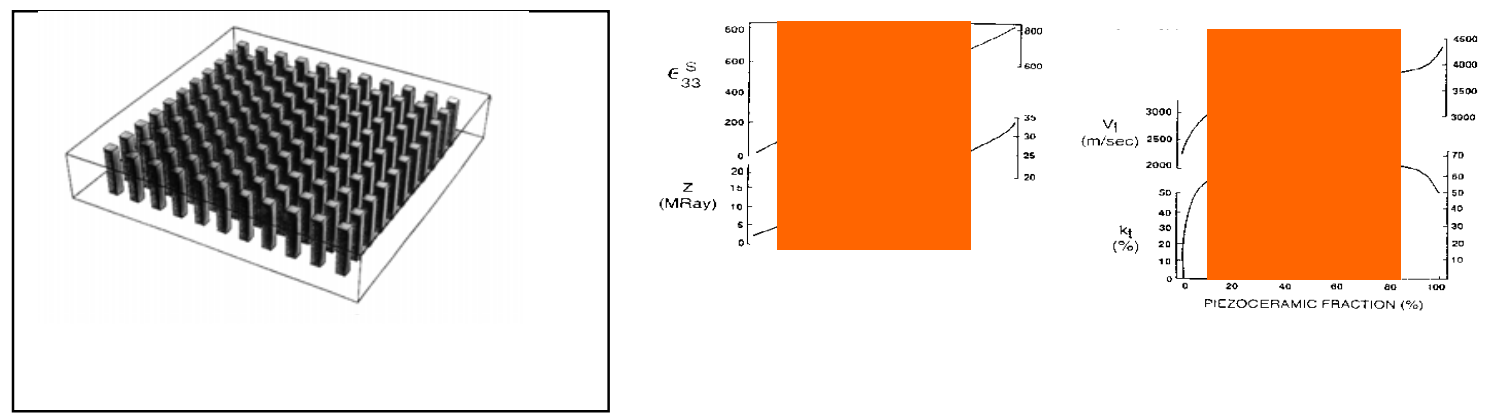

The electroacoustical design is divided in several steps which concern:

The active material: Due to of the anisotropy and the inhomogeneity of materials to inspect, TRLPA transducers must have a good sensitivity level and a broadband. Moreover in order to avoid grating lobes and to obtain a precise beam forming, these transducers require a good cross coupling level between elements, the use of Piezocomposite material allow to improve these parameters.

Imasonic manufactures its own 1-3 structure Piezocomposite material, shown in figure 1.

The piezoelectric ceramic rods are inserted in a polymer material. The ceramic and the resin are chosen according to the characteristics required for the composite material, the geometry of the structure it self can be adapted.

One of the characteristics of the 1-3 structure is that the percentage of ceramic can vary by modifying the size of the rods and their spacing. Figure 2 shows the influence of the percentage of ceramic on the performances of the piezocomposite which are :

- The coupling coefficient kt on which the sensitivity of the array depends

- The dielectric constant e33 on which the electrical impedance depends

- The acoustic impedance Z

- The velocity of propagation in the material on which the frequency for a given thickness depends.

A higher or lower percentage of ceramic gives also different mechanical properties to the composite material. The height of the ceramic rods, which are long compared to their lateral dimensions, favour their vibration according to the thickness mode to the detriment of the radial mode. This results in improved electroacoustical efficiency which gives the array a high level of sensitivity, and high signal to noise ration.

A special piezocomposite material has been designed for the manufacturing of TRLPA transducers, the results make appear a sensitivity improvement between +20 and $+25 \mathrm{~dB}$, compare to the same TRLPA transducers manufactured in ceramic materials.

In addition the natural damping of composite materials allows a relative bandwidth of $70 \%$ to $90 \%$ to be obtained. In the 1-3 composite structure, the isolation of the rods within polymer material enables the transversal propagation of vibrations to be reduced. In the context of Phased array sensors such as TRLPA, this reduction in transversal vibrations limits the propagation of an element signal towards its neighbours (see figure 6).

This independence of the functioning of each channel is fundamental to Phased array technology, as the formation of the beam is based on each element of the transducer being driven using a precise electronic delays.

The level of cross coupling obtained between two elements, is, thanks to this technology less than $-40 \mathrm{~dB}$.

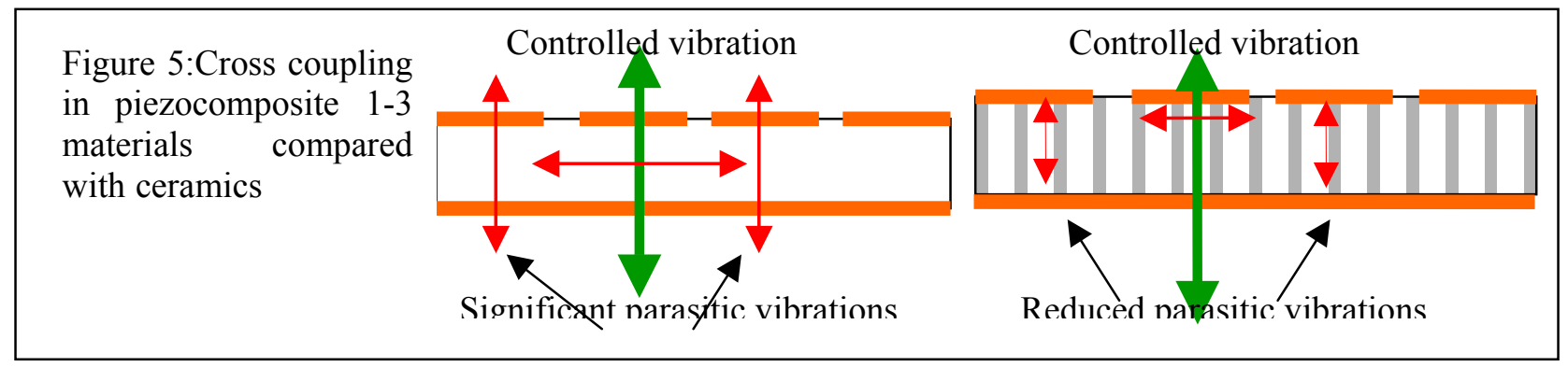


The backing material : The backing material is designed to shorten the pulse length and attenuate the back echo. In reason of the special geometry of the TRLPA transducer (integrated wedges), specially designed backing materials allow interesting compromise with high damping and high attenuation in reduced dimension.

Characterisation and check:

TRLPA transducers are commonly used for critical inspections in sensitive field of activity, requiring a High quality level.

In order to give the best possible quality, different checking are performed at different steps of the manufacturing.

Checking during the manufacturing :

One of the key point is the weld quality, a defect in a weld can damage one or several elements, depending on the location of this weld, repair would be performed easily or not.

In order to avoid such problems, each weld is tested during the manufacturing by applying a high voltage.

Final Characterisation:

The main parameters checked during the final controls are :

Frequency (typically $+/-10 \%$ )

Bandwidth (typically 70 to $90 \%$ )

Cross coupling (typically $<-30 / 40 \mathrm{~dB}$ )

Sensitivity homogeneity (typically $<3 / 4 \mathrm{~dB}$ )

Electrical impedance

Wiring order : This test is performed using the "time of flight" technique, in reason of the integrated wedge which combines roof angle and incidence angle, therefore the distance between successive elements located on a give row and a target located on transducer front face increase regularly. The result is presented in a graph in form of "stairs", allowing easily to see if there is a wiring error.

A probe measurement report is shown below :

\begin{tabular}{|c|c|c|c|c|c|c|c|c|c|}
\hline \multirow{2}{*}{\multicolumn{5}{|c|}{$\begin{array}{l}\text { Eectroacoustic measurement for the XCRn } \\
\text { (Acousticload: 1.5MR IMASONCprocedre: INP413 }\end{array}$}} & \multicolumn{4}{|c|}{ SN04506 } & \multirow[t]{2}{*}{ Operator: } \\
\hline & & & & & & & SIDEF & & \\
\hline \# & $\begin{array}{l}\text { Vpp } \\
(\mathrm{mM})\end{array}$ & \begin{tabular}{c|c|}
$\mathrm{Fc}$ \\
$\left(\mathrm{M} \boldsymbol{H}_{\mathrm{k}}\right)$ \\
\end{tabular} & $\begin{array}{l}\text { Bw } \\
(\%)\end{array}$ & $\begin{array}{l}4 p-20 \mathrm{CB} \\
\text { (ns) }\end{array}$ & \# & $\begin{array}{l}\text { Vpp } \\
(m M) \\
\end{array}$ & $\begin{array}{c}\mathrm{Fc} \\
(\mathrm{M}+\mathrm{t}) \\
\end{array}$ & $\begin{array}{l}\mathrm{Bw} \\
(\%)\end{array}$ & $\begin{array}{l}\text { Lp-200B } \\
\text { (ns) }\end{array}$ \\
\hline 1 & 209.4 & \begin{tabular}{|l|}
1.48 \\
\end{tabular} & 79 & 1440 & 1 & 217.2 & 1.43 & 76 & 2230 \\
\hline 2 & 217.2 & 1.50 & 75 & 1470 & 2 & 217.2 & 1.49 & 73 & 1500 \\
\hline 3 & 226.6 & \begin{tabular}{|l|}
1.51 \\
\end{tabular} & 74 & 1440 & 3 & 209.4 & 1.53 & 73 & 1890 \\
\hline 4 & 2125 & 1.49 & 77 & 1570 & 4 & 206.2 & 1.53 & 76 & 1990 \\
\hline 5 & 207.8 & 1.49 & 79 & 1480 & 5 & 215.6 & 1.53 & 74 & 1890 \\
\hline 6 & 206.2 & 1.48 & 77 & 1600 & 6 & 215.6 & 1.53 & 75 & 4700 \\
\hline 7 & 206.2 & 1.44 & 74 & 1560 & 7 & 215.6 & 1.52 & 76 & 1930 \\
\hline 8 & 223.4 & 1.48 & 76 & 1560 & 8 & 210.9 & 1.53 & 75 & 1920 \\
\hline 9 & 215.6 & 1.50 & 77 & 1510 & 9 & 207.8 & 1.51 & 76 & 1890 \\
\hline 10 & 229.7 & 1.48 & 75 & 1570 & 10 & 207.8 & 1.51 & 75 & 1530 \\
\hline 11 & 214.1 & 1.49 & 78 & 1510 & 11 & 2125 & 1.50 & 78 & 1890 \\
\hline 12 & 237.5 & 1.48 & 78 & 1560 & 12 & 207.8 & 1.53 & 76 & 1490 \\
\hline 13 & 221.9 & 1.49 & 75 & 1500 & 13 & 206.2 & 1.51 & 77 & 1530 \\
\hline 14 & 240.6 & 1.48 & 78 & 1500 & 14 & 217.2 & 1.52 & 75 & 1510 \\
\hline 15 & 221.9 & 1.47 & 77 & 1550 & 15 & 210.9 & 1.50 & 77 & 1520 \\
\hline 16 & 228.1 & 1.48 & 79 & 1550 & 16 & 206.2 & 1.51 & 77 & 1530 \\
\hline
\end{tabular}
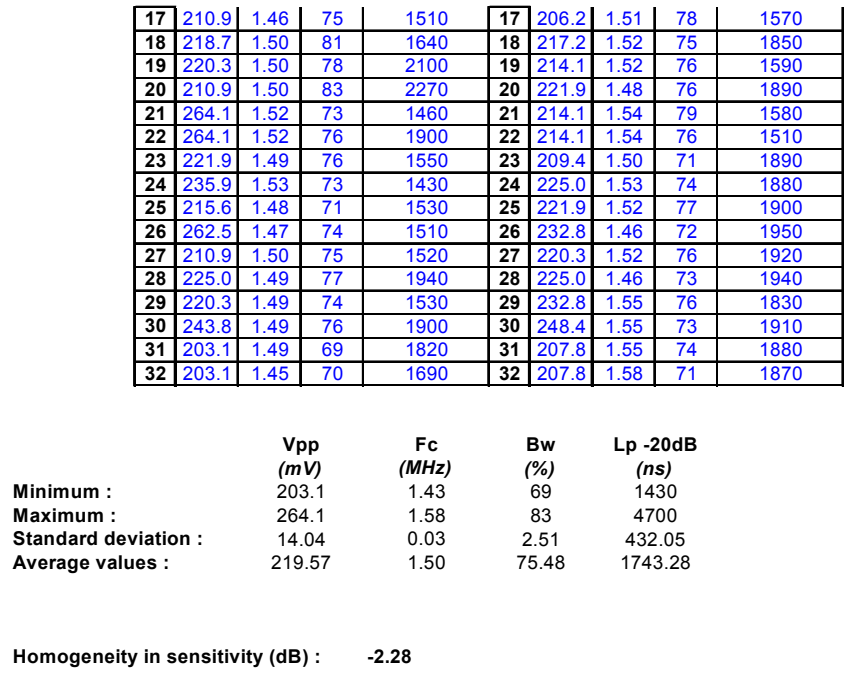

Results: Industrial application of piezocomposite TRLPA for the inspection of austenitic steel:

An "application" consists of a component, where specific flaws have to be detected by mean of specific probes, during a given scanning operation, according to a dedicated UT procedure. The design of an industrial TRLPA will thus depend on

- the component: the thickness, the material (cast, wrought, welds,..) and the geometry of the component, will fix the maximum work frequencies.

- the flaws: the geometry, the position (trough wall) and the orientation (versus the probe displacement) of the defects will give a first idea of the beam shape and angle.

- the scanning technique and the UT procedure: the probe maximum allowed physical displacements (manual or automated with 1 or more degrees of freedom) combined with the required dimensioning 
method will help to define the matrix cutting ( 1 or $2 \mathrm{D}$, ability to realise a sector or linear scan, ability to skew), the acoustic aperture (possibly with focussing) and finally the housing outside dimensions.

The table 1 illustrates a selection of piezocomposite TRLPA capable to detect and characterise cracks by diffraction technique in austenitic welds or bimetallic welds.

\begin{tabular}{|l|c|c|c|c|c|}
\hline $\begin{array}{c}\text { Component } \\
\text { thick. } \\
(\mathrm{mm})\end{array}$ & Component Type & $\begin{array}{c}\text { Freq. } \\
(\mathrm{MHz})\end{array}$ & $\begin{array}{c}\text { Wedge foot } \\
\text { print } \\
\mathrm{L} \times \mathrm{w}(\mathrm{mm})\end{array}$ & $\begin{array}{c}\text { Number of } \\
\text { elements }\end{array}$ & $\begin{array}{c}\text { Number of elements } \\
\text { allowing for lateral skew }\end{array}$ \\
\hline up to 20 & Welds (wrought) & 2.25 & $14 \times 24$ & $/$ & $2 \times(4 \times 8)$ \\
\hline up to 35 & Welds/safe end $)$ & 2.25 & $25 \times 25$ & $2 \times(2 \times 14)$ & $2 \times(3 \times 11)$ \\
\hline up to 50 & Welds/safe end & 2 & $34 \times 34$ & $2 \times(2 \times 16)$ & $2 \times(3 \times 11)$ \\
\hline up to 70 & Safe end $(*)$ & 1.5 & $54 \times 54$ & $2 \times(2 \times 16)$ & $2 \times(3 \times 11)$ \\
\hline 70 & $\begin{array}{c}\text { Primary loop }, \\
\text { petrochem. Reactor }(* *)\end{array}$ & $\begin{array}{c}1 \text { or } \\
1.5\end{array}$ & $54 \times 54$ & $2 \times(2 \times 11)$ & $2 \times(3 \times 11)$ \\
\hline 90 & Primary loop (cast) & 0.75 & $54 \times 54$ & $2 \times(2 \times 11)$ & $2 \times(3 \times 11)$ \\
\hline
\end{tabular}

(*)Example of Application on a SAFE END of a nuclear reactor vessel:

The purpose is the detection and dimensioning of cracks situated between the inside surface (ID) and the outside surface(OD), with automated raster scanning realized from the ID by mean of one probe shown on figure R1

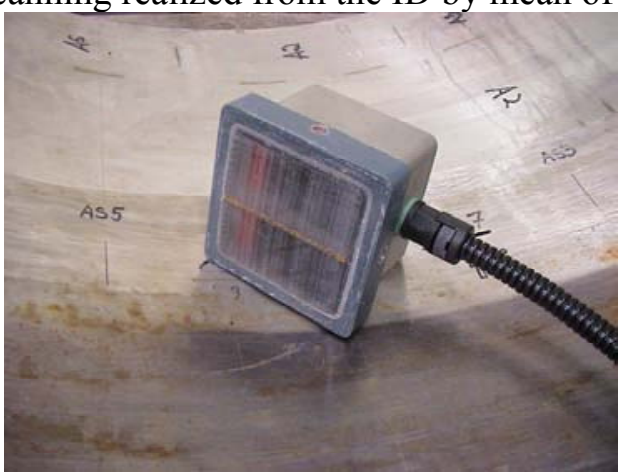

Probe Type: TS55 1.5. 6C1, 2x(2x16) elements, Angle 30 to $90^{\circ}$, foot print $54 \times 54 \mathrm{~mm}$ Figure R1

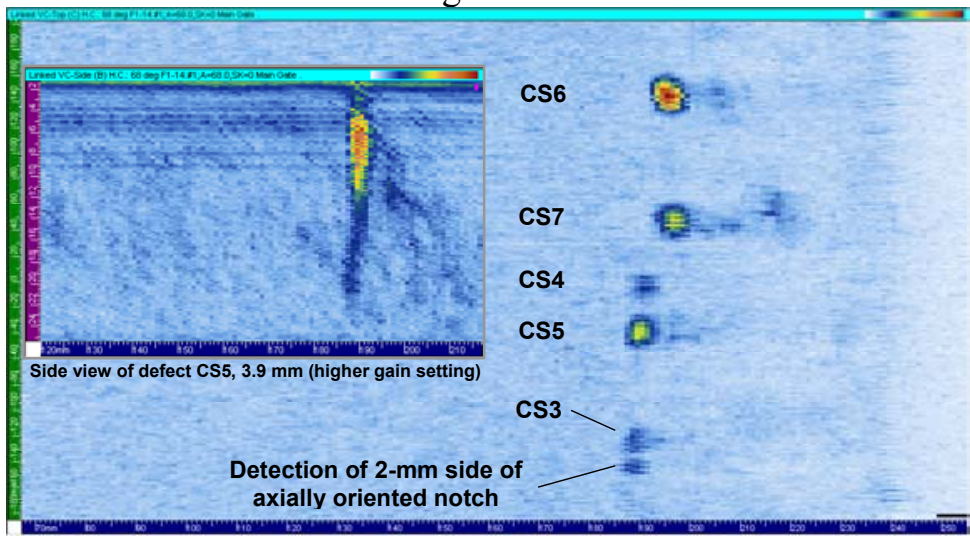

Figure R2

The detection capability on ID circumferentially oriented defects, simulated by EDM notches is illustrated on figure R2 (notch width $=0.3 \mathrm{~mm}$, length $=25 \mathrm{~mm}$, ranging from 1 (CS3) to $15.4 \mathrm{~mm}$ (CS7)). The Signal-to-noise on ID EDM notches ranging from 0.3 to $15.4 \mathrm{~mm}$ depth is shown in figure R3 


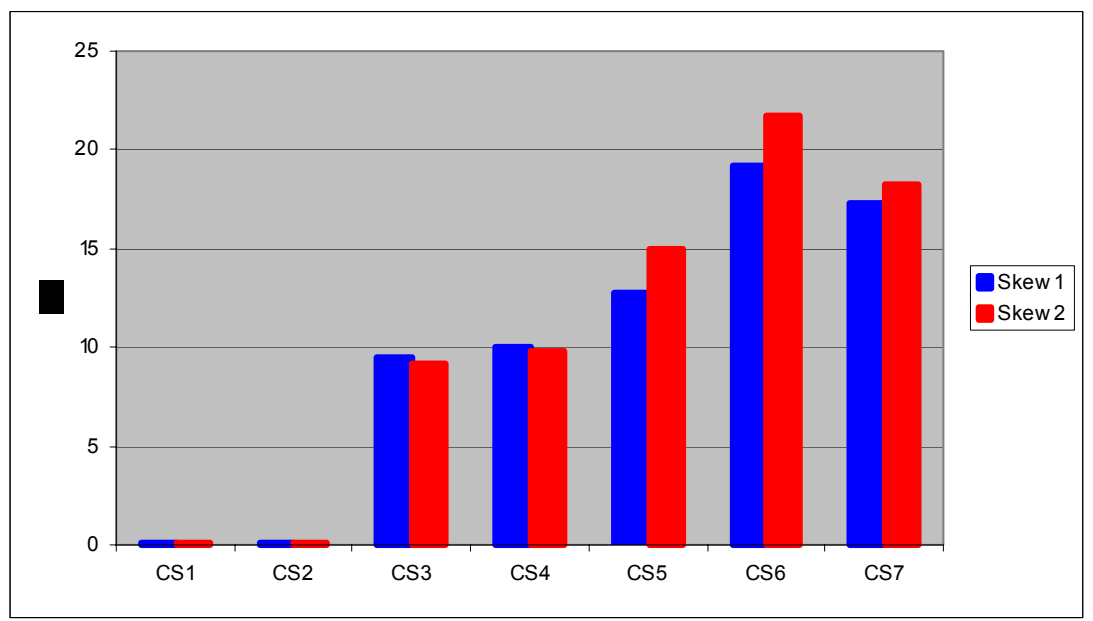

Figure R3

The detection capability on OD circumferentially oriented defects, simulated by EDM notches is illustrated on figure R4 where the tip diffraction is indicated (notch width $=0.3 \mathrm{~mm}$, length $=25 \mathrm{~mm}$, ranging from 1 to 15.4 $\mathrm{mm})$
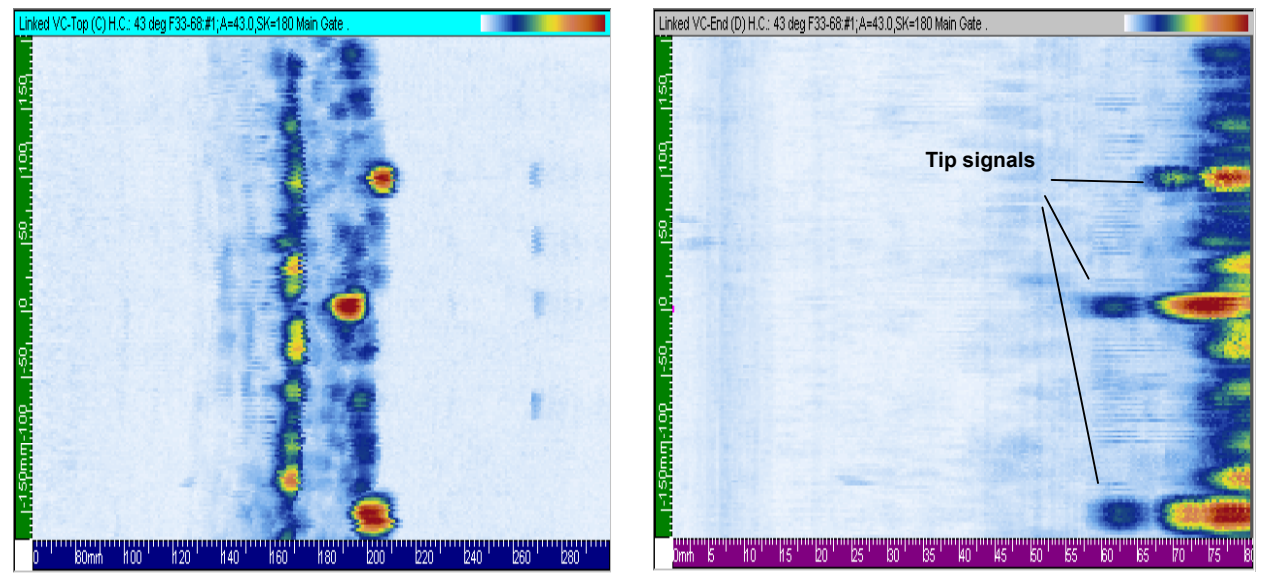

Figure R4

\section{(**) Example of Application on a demethanizer:}

The purpose is the examination, according to the ASMEBPV code section VIII div.1, of a X weld of a demethanizer made of stainless steel SA240 TP304, with a "semi automated one line scanning" operation realised from the ID by mean of two probes, as shown on figure R5

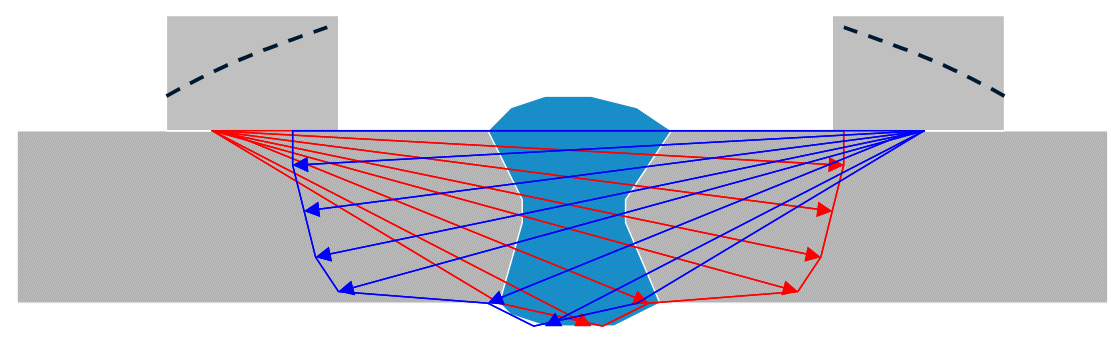


Single line scanning operation (displacement along the weld), volume covered by 2 probes in front of each other, covering the volume by multiple focal laws

Figure R5

All the ASME calibration reflectors (side drilled holes and ID and OD notches) are detected, 100\% of the volume is covered in one operation

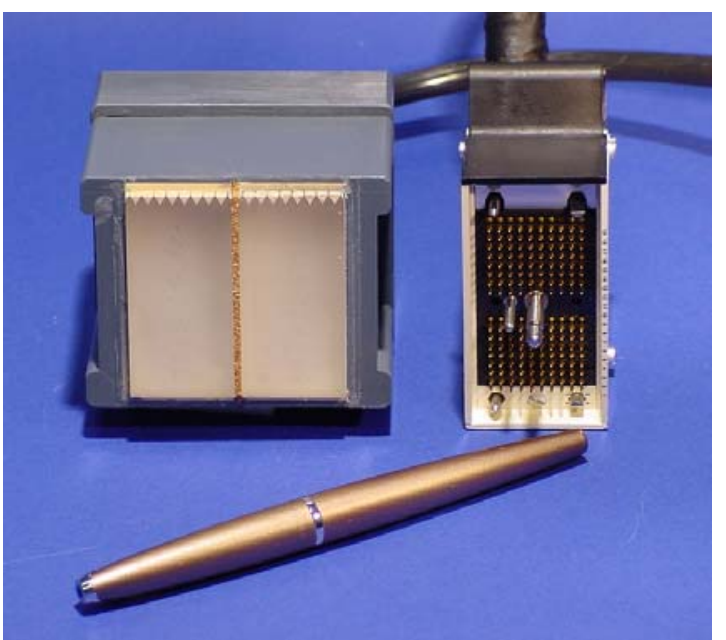

Probe Type: TS45 1 6C1, 2x(2x11) elements, Angle 30 to $85^{\circ}$, foot print $54 x 54 \mathrm{~mm}$ Figure R6

In this example the TRLPA are used in lieu of radiography, resulting in cost reduction and in an increased reliability of the examination.

Conclusions: Piezocomposite TRLPA probes are specially suited for the inspection of stainless steel. They show improved detection and sizing capability compared to single arrays and allow optimised characteristics and increased flexibility compared to standard TRL.

The applications in the industry are various and appreciated because of the cost reduction due to the time saving , or the opportunity to replace radiography. In nuclear environment, the radiation dozes of the operator's can be also drastically limited by the time spent in probe manipulation.

\section{References:}

(1) M. Delaide, G. Maes and D. Verspeelt, "Design and application of Low-Frequency, Twin Side-by-Side, Phased Array Transducers for Improved Ultrasonic Testing Capabilities on Cast Stainless Steel Components", 2nd Int. Conf. on NDE in Relation to Structural Integrity for the Nuclear and Pressured Components, New Orleans, May 24-26, 2000.

(2) Ph. Dumas, G. Fleury and J. Poguet, “ New piezocomposite transducers for Improvement of ultrasonic inspections", QNDE Conf, Bellingham, July 14-19, 2002 